\title{
Pre-activation of the p53 pathway through Nutlin-3a sensitises sarcomas to drozitumab therapy
}

\author{
KATHLEEN I. PISHAS ${ }^{1,2}$, SUSAN J. NEUHAUS ${ }^{1,3},{\text { MARK T. } \text { CLAYER }^{4} \text {, ALAKNANDA ADWAL }}^{1}$, \\ MICHAEL P. BROWN ${ }^{1,5}$, ANDREAS EVDOKIOU ${ }^{1,6}$, DAVID F. CALLEN ${ }^{1}$ and PAUL M. NEILSEN ${ }^{1,2}$ \\ ${ }^{1}$ Centre for Personalised Cancer Medicine, The University of Adelaide; ${ }^{2}$ Sarcoma Research Group, \\ Discipline of Medicine, The University of Adelaide; ${ }^{3}$ Department of Surgery, The University of Adelaide, \\ Royal Adelaide Hospital; ${ }^{4}$ Department of Orthopaedics and Trauma, Royal Adelaide Hospital; \\ ${ }^{5}$ Cancer Clinical Trials Unit, Royal Adelaide Hospital; ${ }^{6}$ Discipline of Surgery, \\ The University of Adelaide, Basil Hetzel Institute, Adelaide, South Australia, Australia
}

Received February 7, 2013; Accepted March 20, 2013

DOI: 10.3892/or.2013.2454

\begin{abstract}
The present study evaluated the efficacy of drozitumab, a human monoclonal agonistic antibody directed against death receptor 5 (DR5), as a new therapeutic avenue for the targeted treatment of bone and soft-tissue sarcomas. The antitumour activity of drozitumab as a monotherapy or in combination with Nutlin-3a was evaluated in a panel of sarcoma cell lines in vitro and human sarcoma patient samples ex vivo. Knockdown experiments were used to investigate the central role of p53 as a regulator of drozitumab cytotoxicity. Pre-activation of the p53 pathway through Nutlin-3a upregulated DR5, subsequently sensitising sarcoma cell lines and human sarcoma specimens to the pro-apoptotic effects of drozitumab. Silencing of p53 strongly decreased DR5 mRNA expression resulting in abrogation of drozitumab-induced apoptosis. Our study provides the first pre-clinical evaluation of combination therapy using p53-activating agents with drozitumab to further sensitise sarcomas to the cytotoxic effects of DR5 antibody therapy.
\end{abstract}

\section{Introduction}

Sarcomas represent a diverse group of heterogeneous mesenchymal neoplasms that affect $\sim 200,000$ individuals worldwide each year. There has been limited improvement in overall 5 -year survival rates for sarcoma patients over the past 30 years, particularly for patients with metastatic disease. As such, the use of molecular-targeted therapies has emerged as a promising new therapeutic approach for the treatment of sarcomas.

Correspondence to: Dr Paul M. Neilsen, Sarcoma Research Group and Centre for Personalised Cancer Medicine, Level 3 Hanson Institute, The University of Adelaide, Frome Road, Adelaide 5000, South Australia, Australia

E-mail:paul.neilsen@health.sa.gov.au

Key words: Nutlin-3a, drozitumab, TP53, sarcoma
Activation of the extrinsic pathway of apoptosis through the use of recombinant ligand and agonistic monoclonal antibodies directed against TRAIL receptors DR4 (TRAIL-R1) and/or DR5 (TRAIL-R2) has been explored as a promising new targeted therapy for numerous types of malignancies. Based on their selective ability to induce apoptosis in a variety of human cancer cell lines and xenografts while sparing normal cells (1), several agents are currently undergoing phase I and II clinical testing both as single agents and in combination with traditional chemotherapies. These include monoclonal agonistic antibodies which specifically target either DR5, drozitumab (Genetech) (2), lexatumumab (Human Genome Sciences) (3-6), conatumumab (Amgen) (7-10), tigatuzumab (humanized IgG1 antibody; Daiichi-Sankyo) and LBY135 [chimeric (mouse/human) IgG1 antibody; Novartis] (11) or DR4, mapatumumab (Human Genome Sciences) (12-17) or both cognate receptors as well as three decoy receptors of recombinant human TRAIL (rhApo2L/TRAIL) (dulanermin, Amgen/Genentech) (18).

Results from early trials have established that DR4 and DR5 agonistic antibodies can be considered safe and are well tolerated with responses not limited to histological subtype. However, the clinical efficacy of these agonistic antibodies as monotherapeutic agents has proven to be quite poor, with only a few patients showing partial or complete responses (19). Out of the 41 evaluable patients who participated in dose escalation phase I clinically testing of drozitumab, partial responses were reported in only 3 patients (chondrosarcoma, colorectal cancer and ovarian cancer) (2). These findings reflect cell culture studies which indicate that only a small subset of sarcoma cell lines are highly sensitive to DR5 agonistic antibodies (20-23).

The poor clinical efficacy of drozitumab as a monotherapy warrants further investigation into combinational therapeutic approaches involving drozitumab with other systemic therapies to synergistically drive tumour regression. Furthermore, additional mechanistic studies are required to completely delineate the fundamental regulators of drozitumab. A broad spectrum of apoptosis regulatory molecules (FLIP, XIAP 
Table I. Primer sequences utilised in this study.

Primer sequence $5^{\prime} \rightarrow 3^{\prime}$

Forward

Reverse

$\begin{array}{ll}\text { PPIG (housekeeping) } & \text { CAGATGCAGCTAGCAAACCGTTTG } \\ \text { DR5 (TRAIL-R2) } & \text { CGCTGCACCAGGTGTGATT } \\ \text { p21 (CDKN1A) } & \text { TGGACCTGGAGACTCTCAGGGTCG } \\ \text { PUMA (BBC3) } & \text { ACGACCTCAACGCACAGTACG } \\ \text { p53 exons 2-4 } & \text { GTGTCTCATGCTGGATCCCCACT } \\ \text { p53 exons 5-6 } & \text { TGCAGGAGGTGCTTACGCATGT } \\ \text { p53 exons 7-9 } & \text { ACAGGTCTCCCCAAGGCGCACT } \\ \text { p53 exon } 10 & \text { GTCAGCTGTATAGGTACTTGAAGTGCAG } \\ \text { p53 exon } 11 & \text { CCTTAGGCCCTTCAAAGCATTGGTCA }\end{array}$

CTCTTCAGTAGCACTTTCGGAATCAGAGG GTGCCTTCTTCGCACTGACA TTAGGGCTTCCTCTTGGAGAAGATC TCCCATGATGAGATTGTACAGGAC GGATACGGCCAGGCATTGAAGT CCTTAACCCCTCCTCCCAGAGAC TTGAGGCATCACTGCCCCCTGAT TGGCAGCTGAGCTAGACCTCG GTGCTTCTGACGCACACCTATTGCAAG

and $\mathrm{Bcl}-\mathrm{X}_{\mathrm{L}}$ ) and signalling pathways (NF- $\mathrm{BB}$ and $\mathrm{Akt}$ ) are believed to confer resistance; however, little is known concerning the influence of proteins which sensitise tumour cells to drozitumab therapy, apart from DR5 itself. The protein and mRNA expression levels of DR5 and DR4 in sarcoma cell lines have been extensively documented in literature. Notably, resistance to TRAIL-mediated apoptosis is not associated with differential expression of TRAIL-receptors between sensitive and resistant sarcoma cell lines $(24,25)$. As DR5 has been shown to be a transcriptional target of p53 (26), this study assessed the role of p53 in mediating sensitivity to drozitumab in sarcoma cell lines and human sarcoma patient material. As expected, knockdown of p53 ablated drozitumab-induced apoptosis in vitro. Furthermore, pre-activation of the p53 pathway through Nutlin-3a (p53-MDM2 antagonist) enhanced the cytotoxic effects of drozitumab both in vitro and ex vivo. Our study provides the first pre-clinical evidence that preactivation of the p53 pathway in conjunction with drozitumab will potentially provide an effective therapeutic means to maximise the apoptotic response from both the extrinsic and intrinsic pathway for the treatment of sarcomas.

\section{Materials and methods}

Cell culture. Osteosarcoma (Saos-2, U20S) and Ewing's sarcoma (SK-ES1, RD-ES) cell lines were purchased from American Type Tissue Culture (ATCC, Manassas, VA, USA). Additional Ewing's sarcoma cell lines CADO-ES1, STA-ET1, SK-N-MC, TC252, VH-64, WE-68 were kindly supplied by J. Sonnemann (Department of Pediatric Haematology and Oncology, University Children's Hospital, Jena, Germany), P. Ambros (Children's Cancer Research Institute, St. Anna Children's Hospital, Vienna, Austria), V. Russo (Murdoch Children's Research Institute, Royal Children's Hospital, Victoria, Australia), G. Hamilton (Department of Surgery, University of Vienna, Austria) and F. van Valen (Department of Orthopaedic Surgery, Westfälische Wilhelms University, Germany). Cell lines were cultured as previously described (27).

Cell viability assays. Cells were seeded in 96-well microtiter plates at a density of $3 \times 10^{4}$ cells/well in the presence of droz- itumab + anti-Fc $\gamma$ at the indicated concentrations for $24 \mathrm{~h}$. Drozitumab (a kind gift from Dr Avi Ashkenazi, Genentech Inc., South San Francisco, CA, USA), was cross-linked with anti-human IgG Fc $\gamma$ antibody (Jackson ImmunoResearch Laboratories, Inc., West Grove, PA, USA) as previously described (28). For synergy experiments, cells were pre-treated with Nutlin-3a (Cayman Biochemicals, Ann Arbor, MI, USA) for $24 \mathrm{~h}$ prior to the addition of drozitumab + anti-Fc $\gamma$. Cells were harvested and processed as previously described (27). The viability of harvested cells was determined using 7-aminoactinomycin-D staining and processed on a FACSCalibur flow cytometer (Becton-Dickinson Immunocytometry Systems, Franklin Lakes, NJ, USA).

RNA interference. Cell lines with silenced expression of $\mathrm{p} 53$ were generated using the pGIPZ lentiviral shRNAmir system (Open Biosystems) as previously described (29). Briefly, HEK-293T cells were seeded at 50\% confluency and transfected with either a non-silencing scramble control (RHS4346) or shRNA directed against human p53 (V2LHS217) using Trans-Lentiviral packaging mix according to the manufacturer's protocol (Thermo Fisher Scientific, Waltham, MA, USA). Forty-eight hours post-transfection, growth medium containing lentivirus particles was filtered and added to recipient TC252 cells seeded at 50\% confluency. Polyclonal populations of transduced cells were generated through subsequent puromycin selection

Western blot analysis. Western blot analysis was performed as previously described (30). Protein extracts were resolved by SDS polyacrylamide gel electrophoresis on $8 \%$ polyacrylamide gels and incubated with anti-p53 DO-1 (1:1,000, Santa Cruz Biotechnology, Santa Cruz, CA, USA). Anti- $\beta$-actin (1:2,000; Sigma Aldrich, St. Louis, MO, USA) was used as an internal loading control.

Real-time PCR. Total RNA was extracted using RNeasy Mini kit (Qiagen), using On-Column RNase-free DNase digestion according to the manufacturer's instructions. cDNAs were generated and real-time PCR reactions were processed and normalised as previously described (31). Primer sequences are listed in Table I. 


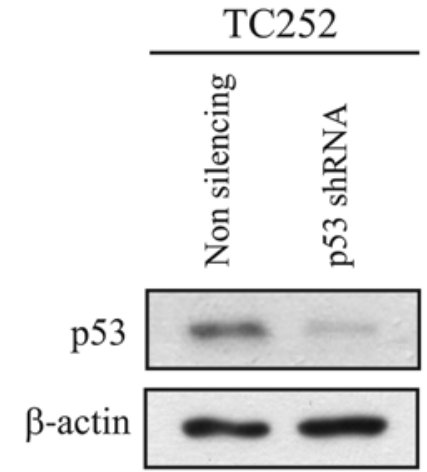

Figure 1. Knockdown of p53 in TC252 cells. Western blot analysis of p53 expression in TC252 non-silencing and p53 shRNA cell lines. Total $\beta$-actin level was used as a protein loading control.

Explant system. The ex vivo sarcoma tissue explant system was adapted from methods previously described (32). Briefly sarcoma tissue from patients previously not exposed to neoadjuvant therapy was collected immediately following surgical resection and treated with the following: vehicle control (DMSO), Nutlin-3a $(10 \mu \mathrm{M})$, drozitumab as a monotherapy $(200 \mathrm{ng} / \mathrm{ml})$ and in combination with Nutlin-3a $(10 \mu \mathrm{M})$ for $48 \mathrm{~h}$. For synergy experiments, explants were pre-treated with Nutlin-3a for $24 \mathrm{~h}$ prior to the addition of drozitumab + anti Fc $\gamma$. Paraffin-embedded sections were subjected to immunohistochemical (IHC) analysis for activated-caspase 3 (ab4051, 1:100; Abcam, Cambridge, UK). IHC analysis was adapted from methods previously described (32). Sequencing of exons 2-11 of the TP53 gene was conducted to confirm the p53 status of the sarcoma tissue.

Ethical approval. This study was performed with the approval of the Royal Adelaide Hospital Human Research Ethics Committee (protocol \#100505). The research conducted throughout is compliant with the Helsinki Declaration and adheres to the guidelines stated by the National Health and Medical Research Council (NHMRC) of Australia.

Statistics. Combination index (CI) values were used to determine the effects of drozitumab on cell viability in the presence and absence of Nutlin-3a, as previously described (33). A CI of 1 indicates an additive effect; $>1$, an antagonistic effect; and $<1$, a synergistic effect.

\section{Results and Discussion}

In an effort to define the role of p53 in the cytotoxic response of sarcoma cells to drozitumab, lentiviral-based delivery of shRNAs targeting p53 (or non-targeting control shRNAs) were delivered into the wild-type p53 Ewing's sarcoma cell line TC252. Knockdown of p53 resulted in effective ablation of p53 protein levels (Fig. 1). The sensitivity of these TC252 derivatives expressing either p53 shRNA or control shRNA to drozitumab was subsequently determined. In the control (non-silencing shRNA) cell line derivative, drozitumab induced a dose-dependent increase in cytotoxicity, with an $\mathrm{IC}_{50}$ of $54 \mathrm{ng} / \mathrm{ml}$ (Fig. 2A). In contrast, silencing of p53 significantly ablated the ability of drozitumab to induce apoptosis

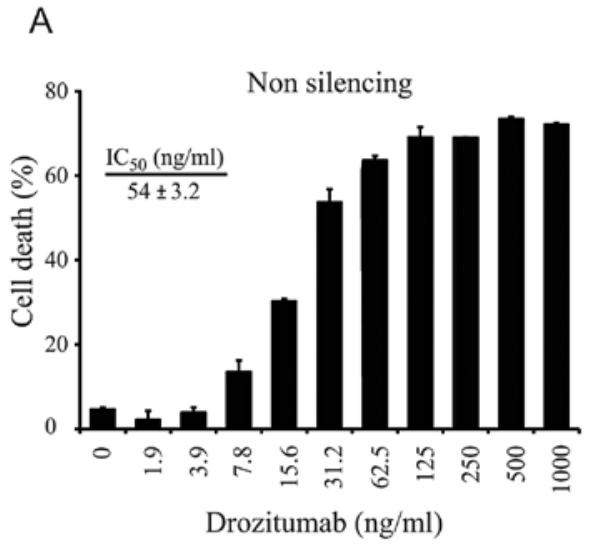

B

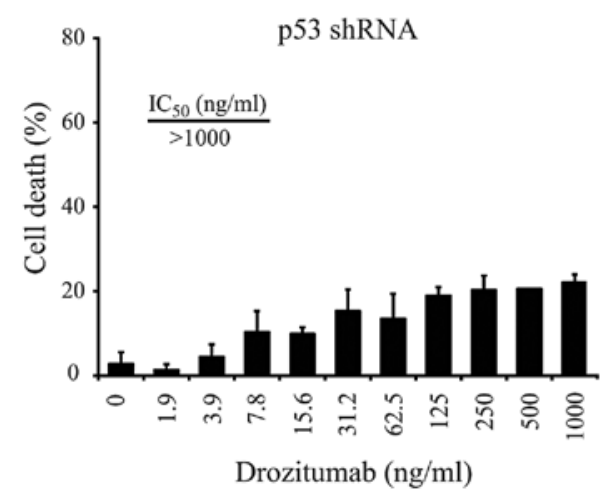

Figure 2. Knockdown of p53 ablates the cytotoxic effects of drozitumab. (A) TC252 non-silencing and (B) p53 shRNA cell lines were treated with increasing concentrations of drozitumab $(0-1,000 \mathrm{ng} / \mathrm{ml})$ for $24 \mathrm{~h}$. Cell viability was determined by 7AAD staining and analysed by flow cytometry (triplicate reactions). The percentage of cell death (means \pm SE) for each treatment is shown.

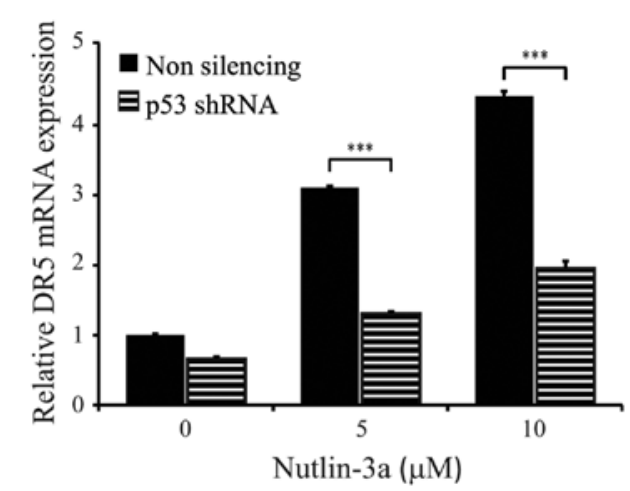

Figure 3. DR5 expression is p53 dependent. TC252 non-silencing and p53 shRNA cell lines were treated with Nutlin-3a $(0,5$ and $10 \mu \mathrm{M})$ for $8 \mathrm{~h}$. RNA was extracted from cells, with relative target DR5 mRNA expression levels determined by RT-PCR analysis. Data represent means \pm SE from triplicate reactions. ${ }^{* * *} \mathrm{P}<0.0001$, statistical significance as determined by unpaired two-tailed t-test.

$\left(\mathrm{IC}_{50}>1,000 \mathrm{ng} / \mathrm{ml}\right)$. Even at the maximum concentration tested $(1,000 \mathrm{ng} / \mathrm{ml})$, drozitumab was only able to induce $22 \%$ cell death (Fig. 2B). As DR5 is a p53-regulated gene (26), we wished to confirm that this observed ablation in drozitumabinduced cytotoxicity was attributed to reduced DR5 expression. Activation of the p53 pathway was achieved through the use 
A

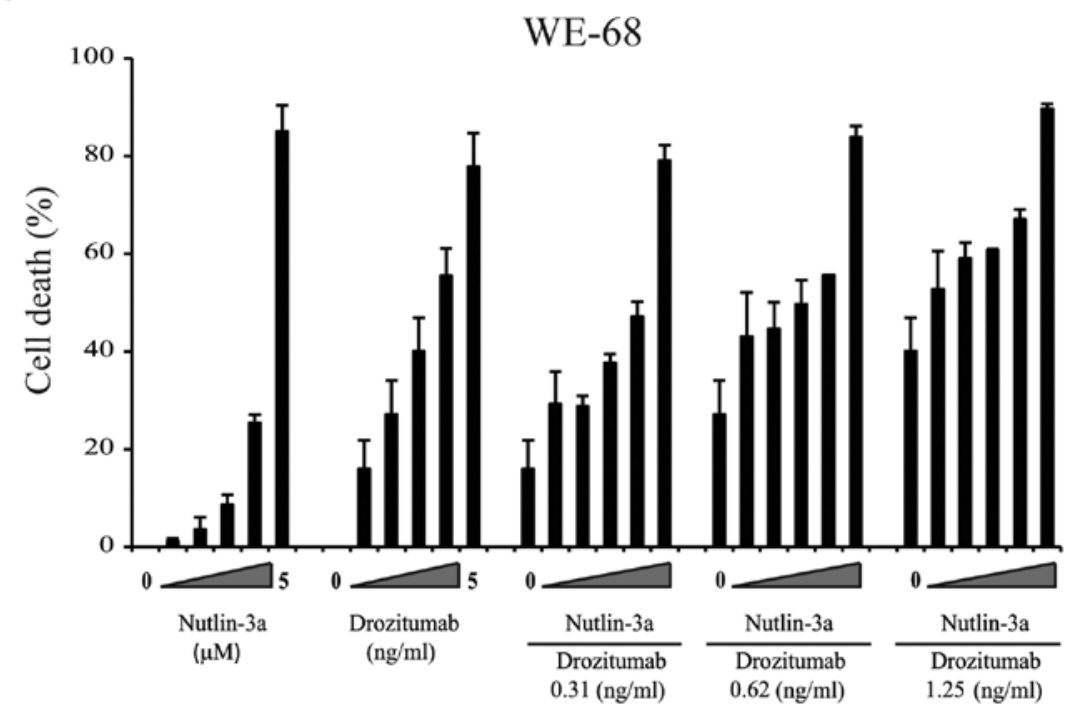

B

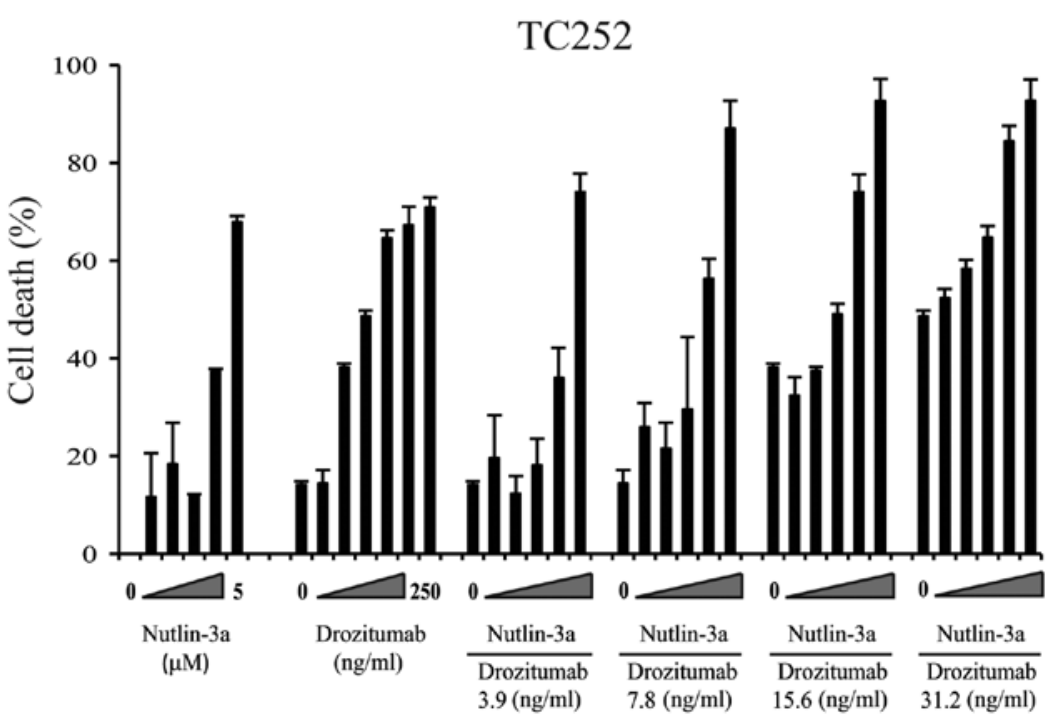

Figure 4. Nutlin-3a enhances the apoptotic response to drozitumab. (A) WE-68 and (B) TC252 cells were pre-treated with Nutlin-3a (0-5 $\mu$ M) for 24 h, prior to the addition of drozitumab (concentrations as indicated) for a further $24 \mathrm{~h}$. Cell viability was determined by 7AAD staining and analysed by flow cytometry. The percentage of cell death for each treatment is shown.

of the non-genotoxic agent Nutlin-3a. Indeed, significant DR5 upregulation was observed following Nutlin-3a treatment of control (non-silencing shRNA) TC252 cells. In contrast, TC252 cells expressing the p53-specific shRNA were associated with a significantly diminished capacity to upregulate DR5 upon p53 activation $(\mathrm{P}<0.0001)$ (Fig. 3). These results suggest that p53-induced expression of DR5 is required for conferring drozitumab sensitivity.

As Nutlin-3a treatment upregulated DR5 mRNA expression in a p53-dependent manner, we sought to determine whether pre-activation of the p53 pathway with Nutlin-3a augments drozitumab induced apoptosis in vitro. WE- 68 or TC252 sarcoma cell lines were pre-treated with Nutlin-3a for $24 \mathrm{~h}$ before the addition of drozitumab for an additional $24 \mathrm{~h}$. This combined dose and schedule of Nutlin-3a and drozitumab resulted in synergistic cell death, with maximum combination indices of $\sim 0.5$ in both cell lines (Fig. 4, Table II). Furthermore, synergism between these two agents was only
Table II. Combination index (CI) values.

\begin{tabular}{cccc}
\hline & & \multicolumn{2}{c}{ In combination with Nutlin-3a } \\
\cline { 3 - 4 } Cell line & $\begin{array}{c}\text { Drozitumab } \\
\mathrm{IC}_{50}(\mathrm{ng} / \mathrm{ml})\end{array}$ & $\begin{array}{c}\text { Drozitumab dose } \\
(\mathrm{ng} / \mathrm{ml})\end{array}$ & $\begin{array}{c}\text { Combination } \\
\text { index }\end{array}$ \\
\hline WE-68 & \multirow{2}{*}{$1.5 \pm 0.8$} & 0.31 & 0.62 \\
& & 0.62 & 0.54 \\
$\mathrm{TC} 252$ & \multirow{2}{*}{$40.7 \pm 2.2$} & 1.25 & 0.87 \\
& & 3.9 & 0.96 \\
& & 7.8 & 0.57 \\
& & 15.6 & 0.52 \\
& & 31.2 & 0.79 \\
\hline
\end{tabular}

$\mathrm{CI}<1, \mathrm{CI}=1$ and $\mathrm{CI}>1$ indicate synergism, additive effect and antagonism, respectively. 


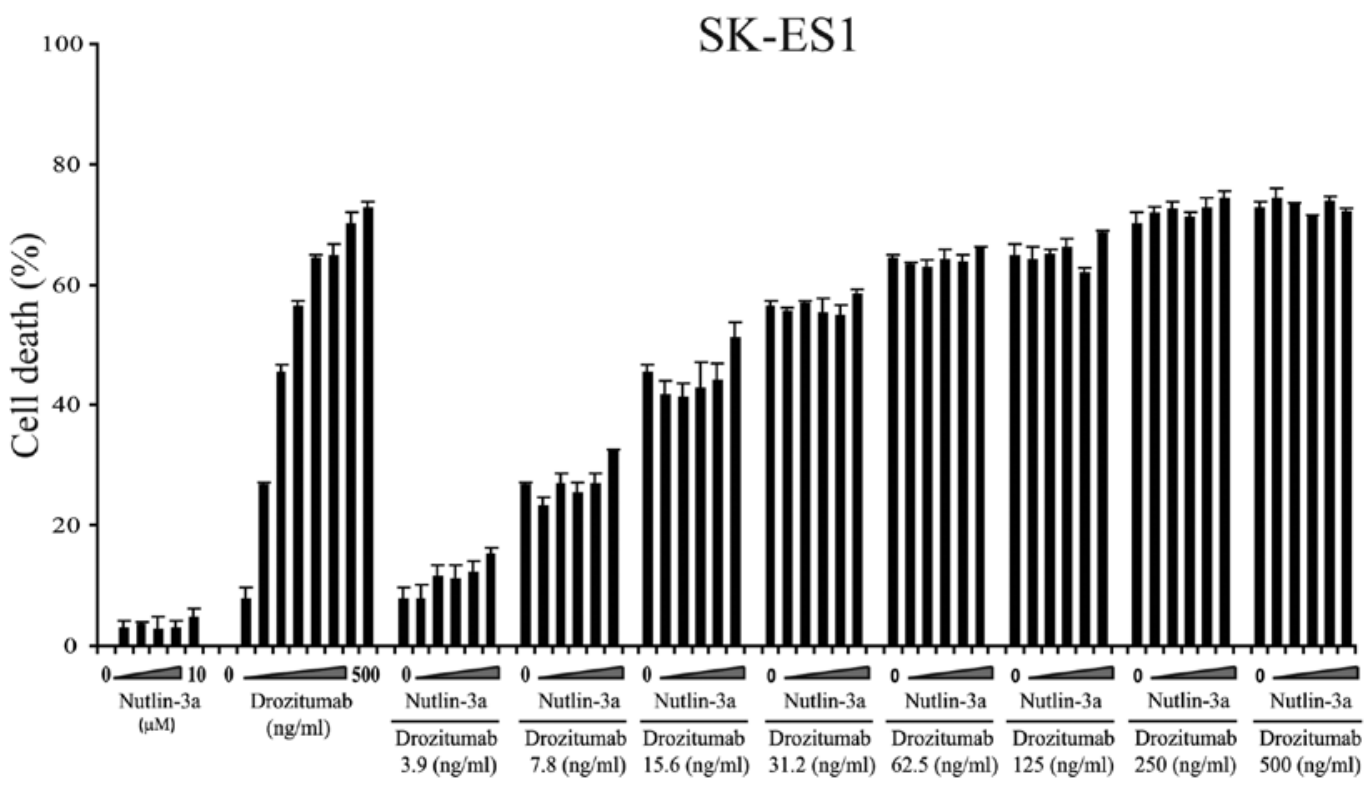

Figure 5. Synergy between Nutlin-3a and drozitumab is dependent on p53. Mutant p53 cell line SK-ES1 was pre-treated with Nutlin-3a (0-10 $\mu \mathrm{M})$, prior to the addition of drozitumab $(0-500 \mathrm{ng} / \mathrm{ml})$ for a further $24 \mathrm{~h}$. Cell viability was determined by 7AAD staining and analysed by flow cytometry. The percentage of cell death for each treatment is shown.

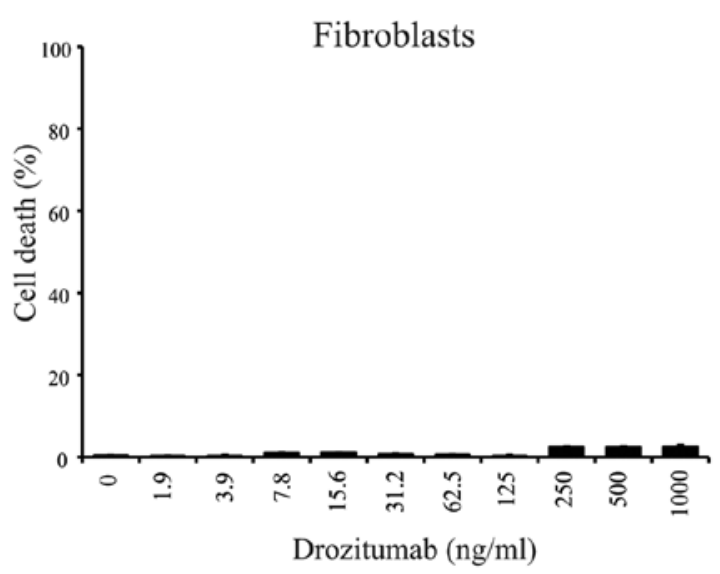

Figure 6. Drozitumab treatment does not affect normal cells. Normal human fibroblasts were treated with increasing concentrations of drozitumab $(0-1,000 \mathrm{ng} / \mathrm{ml})$ for $24 \mathrm{~h}$. Cell viability was determined by 7AAD staining and analysed by flow cytometry. The percentage of apoptotic cells is shown.

achieved in the wild-type p53 cell lines WE-68 and TC-252 but not in the mutant p53-expressing cell line SK-ES1 (Fig. 5). Importantly, the viability of normal human fibroblasts was unaffected following exposure to drozitumab at these concentrations (Fig. 6).

We subsequently investigated whether Nutlin-3a augments the apoptotic response of drozitumab in human sarcoma patient samples ex vivo. Sarcoma tissues from 3 patients were collected immediately following surgical resection, dissected in $1-\mathrm{mm}^{3}$ pieces and pre-treated using an ex vivo tissue explant system with Nutlin-3a $(10 \mu \mathrm{M})$ for $24 \mathrm{~h}$ prior to the addition of drozitumab $(200 \mathrm{ng} / \mathrm{ml})$ for a subsequent $24 \mathrm{~h}$. An increased level of apoptosis following this combination treatment was achieved in 1 of the 3 patients (patient 1; malignant fibrous histiocytoma) (Fig. 7A). This response was associated with a significant upregulation of DR5 receptor expression
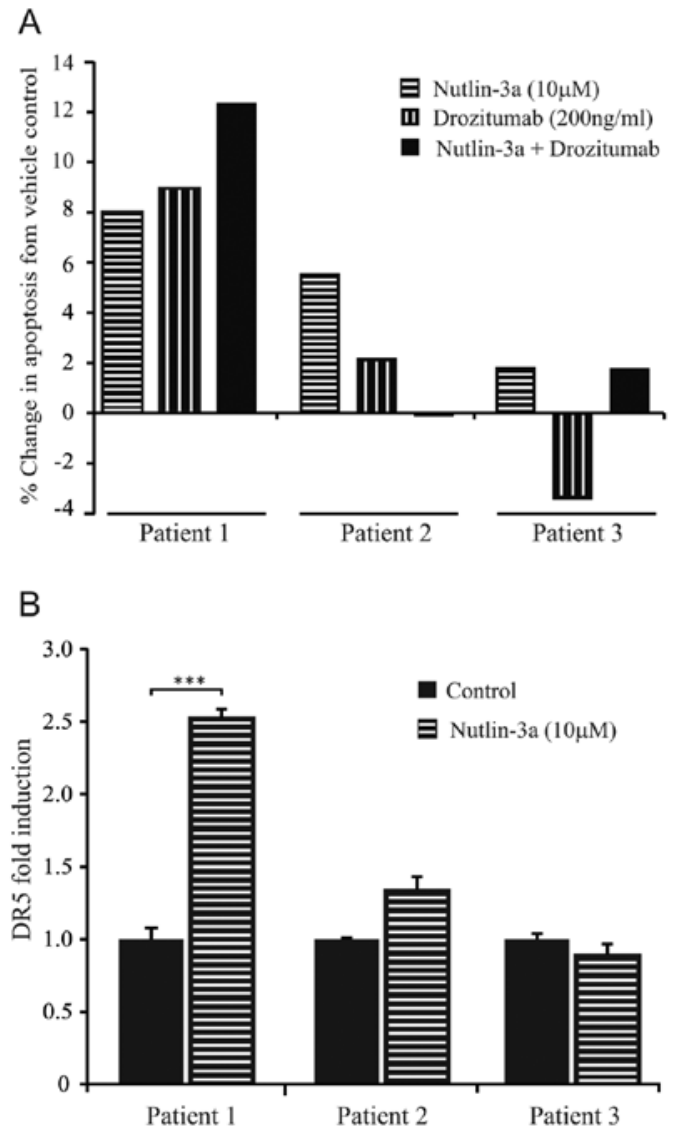

Figure 7. Nutlin-3a synergises with drozitumab in human sarcoma patient samples ex vivo. (A) Sarcoma tissue from 3 patients were treated as follows: Nutlin-3a alone (10 $\mu \mathrm{M}, 48 \mathrm{~h})$, drozitumab alone (200 ng/ml, $24 \mathrm{~h})$, or pretreated with Nutlin-3a $(10 \mu \mathrm{M})$ for $24 \mathrm{~h}$ before the addition of drozitumab (200 ng/ml) for an additional $24 \mathrm{~h}$. The percent change in apoptotic cells from the vehicle control was evaluated by immunohistochemical analysis for activated-caspase 3. (B) Relative DR5 mRNA expression levels (fold-induction) from sarcoma explants treated with vehicle control or Nutlin-3a $(10 \mu \mathrm{M})$ for $24 \mathrm{~h}$ were determined by RT-PCR analysis. ${ }^{* * *} \mathrm{P}<0.0001$, statistical significance. Data represent means $\pm \mathrm{SE}$ from triplicate reactions. 
A

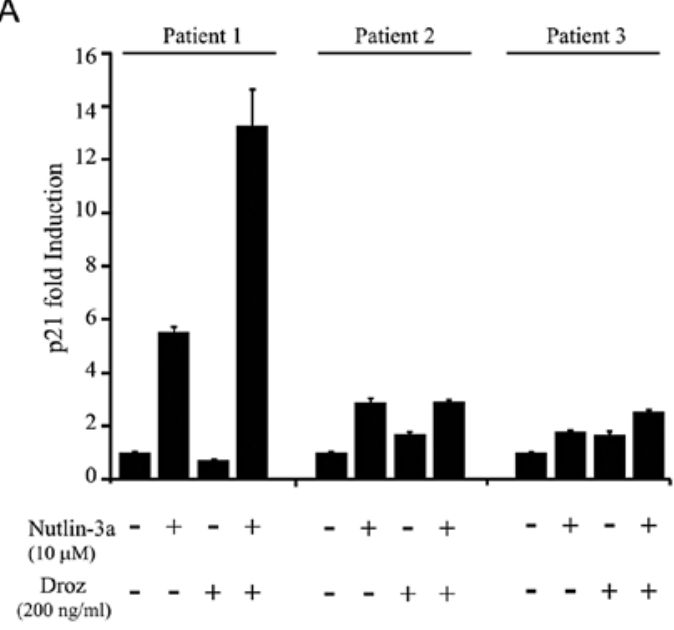

B

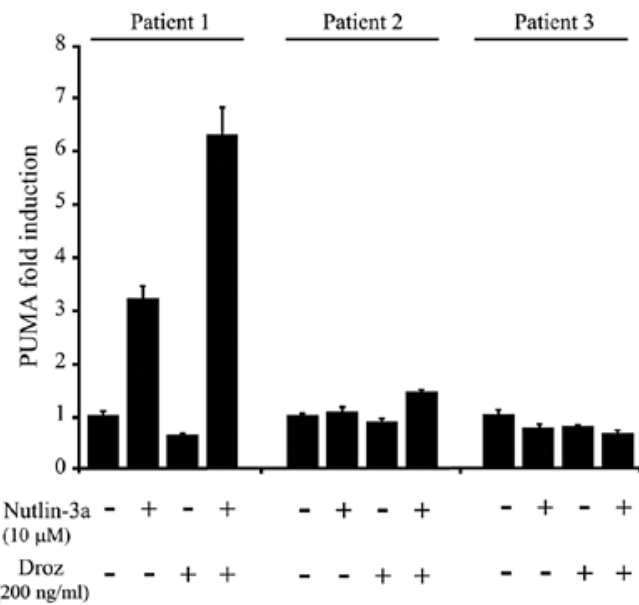

Figure 8. Induction of target genes following Nutlin-3a/drozitumab treatment. Sarcoma tissue from 3 patients were treated as follows: Nutlin-3a alone (10 $\mu \mathrm{M}, 48 \mathrm{~h})$, drozitumab (Droz) alone $(200 \mathrm{ng} / \mathrm{ml}, 24 \mathrm{~h})$, or pre-treated with Nutlin-3a $(10 \mu \mathrm{M})$ for $24 \mathrm{~h}$ prior to the addition of drozitumab $(200 \mathrm{ng} / \mathrm{ml})$ for an additional $24 \mathrm{~h}$. (A) Relative p21 and (B) PUMA mRNA expression levels (fold-induction) from sarcoma explants were determined by RT-PCR analysis. Data represent means $\pm \mathrm{SE}$ from triplicate reactions.

$(\mathrm{P}<0.0001)$ following Nutlin-3a treatment, thus, providing a plausible mechanism for this enhanced apoptotic response (Fig. 7B). In contrast, sarcomas from patients 2 and 3 showed neither an increase in drozitumab efficacy upon pre-treatment with Nutlin-3a, nor DR5 upregulation following Nutlin-3a pre-treatment. Furthermore, the ability of this combination treatment to induce the expression of other verified p53 target genes (p21 and PUMA) was also only observed in the responding patient (patient 1) (Fig. 8). Therefore, the ability of p53 to stimulate DR5 expression in sarcoma tissues is a key factor that contributes towards the synergistic effects between p53 activators and drozitumab.

To further investigate the role of p53 in modulating susceptibility of sarcoma cell lines to the cytotoxic effects of drozitumab, viability assays were carried out on a panel of 10 sarcoma cell lines with varying p53 statuses in vitro. Notably, there was no significant correlation between p53 status of the sarcoma cell lines and sensitivity to drozitumab (Table III), suggesting that p53 status alone is not an indispensable deter-
Table III. Sensitivity of sarcoma cell lines to drozitumab.

\begin{tabular}{lclc}
\hline Cell line & Histology & TP53 status & $\begin{array}{c}\text { Drozitumab } \mathrm{IC}_{50} \\
(\mathrm{ng} / \mathrm{ml})\end{array}$ \\
\hline WE-68 & ES & Wild-type & $6.0 \pm 0.1$ \\
SK-N-MC & ES & Null & $6.8 \pm 0.1$ \\
STA-ET1 & pPNET & Wild-type & $7.7 \pm 2.2$ \\
RD-ES & ES & Mutant & $37.2 \pm 5.5$ \\
TC252 & ES & Wild-type & $54.9 \pm 3.2$ \\
VH-64 & ES & Wild-type & $65.2 \pm 1.7$ \\
SK-ES1 & ES & Mutant & $214.1 \pm 19.2$ \\
CADO-ES1 & ES & Wild-type & $>1,000$ \\
U2OS & OS & Wild-type & $>1,000$ \\
Saos-2 & OS & Null & $>1,000$
\end{tabular}

ES, Ewing's sarcoma; pPNET, peripheral primitive neuroectodermal tumour; OS, osteosarcoma; $\mathrm{IC}_{50}$, concentration of drozitumab required to cause $50 \%$ cell death.

minant for driving drozitumab sensitivity. In particular, two of the wild-type p53 sarcoma cell lines (CADO-ES1 and U2OS) were completely resistant to drozitumab $\left(\mathrm{IC}_{50}>1,000 \mathrm{ng} / \mathrm{ml}\right)$. However, it must be noted that the Ewing's sarcoma cell line CADO-ES1 is deficient in caspase- 8 expression, an essential protein required for the initiation of the extrinsic pathway of apoptosis (34). In summary, although p53 may play a critical role in drozitumab sensitivity in sarcomas that have retained a wild-type p53, our data suggest that the p53 status of cell lines alone is not enough to predict drozitumab cytotoxicity, most likely due to secondary genetic alterations in the tumour that drive fundamental defects in the apoptotic pathway. Thus, further mechanistic studies are required to define other factors that can influence the susceptibility of sarcomas to drozitumab-mediated apoptosis. Collectively, our results justify further pre-clinical investigations of therapeutic regimes that combine DR5 agonists with p53 activators as a new means to amplify crosstalk signals from both the extrinsic and intrinsic pathways of apoptosis for the targeted treatment of sarcoma.

\section{Acknowledgements}

This study was supported in part by the ASSG Sarcoma Research Award funded through the Rainbows for Kate Foundation in memory of Tom Wood. We wish to acknowledge financial support from the Florey Medical Research Foundation, Royal Adelaide Hospital Clinical Project Grant, Cancer Australia (APP1034715) and the Cure Cancer Australia Foundation.

\section{References}

1. Ashkenazi A, Pai RC, Fong S, et al: Safety and antitumor activity of recombinant soluble Apo2 ligand. J Clin Invest 104: 155-162, 1999.

2. Camidge DR, Herbst RS, Gordon MS, et al: A phase I safety and pharmacokinetic study of the death receptor 5 agonistic antibody PRO95780 in patients with advanced malignancies. Clin Cancer Res 16: 1256-1263, 2010. 
3. Wakelee HA, Patnaik A, Sikic BI, et al: Phase I and pharmacokinetic study of lexatumumab (HGS-ETR2) given every 2 weeks in patients with advanced solid tumors. Ann Oncol 21: 376-381, 2010.

4. Plummer R, Attard G, Pacey S, et al: Phase 1 and pharmacokinetic study of lexatumumab in patients with advanced cancers. Clin Cancer Res 13: 6187-6194, 2007.

5. Merchant MS, Chou AJ, Price A, Geller JI, Tsokos M, Graham C, Charles A, Meyers PA and Mackall C: Lexatumumab: results of a phase I trial in pediatric patients with advanced solid tumors. J Clin Oncol 18 (Suppl: 15): 9500, 2010.

6. Sikic BI, Wakelee HA, von Mehren M, Lewis N, Calvert AH Plummer ER, Fox NL, Howard T, Jones SF and Burris HA: A phase Ib study to assess the safety of lexatumumab, a human monoclonal antibody that activates TRAIL-R2, in combination with gemcitabine, pemetrexed, doxorubicin or FOLFIRI. J Clin Oncol 25 (Suppl 18): 14006, 2007.

7. Demetri GD, Le Cesne A, Chawla SP, et al: First-line treatment of metastatic or locally advanced unresectable soft tissue sarcomas with conatumumab in combination with doxorubicin or doxorubicin alone: a phase I/II open-label and double-blind study. Eur J Cancer 48: 547-563, 2012.

8. Doi T, Murakami H, Ohtsu A, et al: Phase 1 study of conatumumab, a pro-apoptotic death receptor 5 agonist antibody, in Japanese patients with advanced solid tumors. Cancer Chemother Pharmacol 68: 733-741, 2011.

9. Herbst RS, Kurzrock R, Hong DS, et al: A first-in-human study of conatumumab in adult patients with advanced solid tumors. Clin Cancer Res 16: 5883-5891, 2010.

10. Chawla SP, Tabernero J, Kindler HL, Chiorean EG, LoRusso P, Hsu M, Haddad V, Bach BA and Baselga J: Phase I evaluation of the safety of conatumumab (AMG 655) in combination with AMG 479 in patients (pts) with advanced, refractory solid tumors. J Clin Oncol 28: 3102, 2010.

11. Sharma S, de Vries EG, Infante JR, Oldenhuis C, Chiang L, Bilic S, Goldbrunner M, Scott JW and Burris HA III: Phase trial of LBY135, a monoclonal antibody agonist to DR5, alone and in combination with capecitabine in advanced solid tumors. J Clin Oncol 26: 3538, 2008.

12. Hotte SJ, Hirte HW, Chen EX, et al: A phase 1 study of mapatumumab (fully human monoclonal antibody to TRAIL-R1) in patients with advanced solid malignancies. Clin Cancer Res 14 3450-3455, 2008.

13. Leong S, Cohen RB, Gustafson DL, et al: Mapatumumab, an antibody targeting TRAIL-R1, in combination with paclitaxel and carboplatin in patients with advanced solid malignancies: results of a phase I and pharmacokinetic study. J Clin Oncol 27: 4413-4421, 2009.

14. Mom CH, Verweij J, Oldenhuis CN, et al: Mapatumumab, a fully human agonistic monoclonal antibody that targets TRAIL-R1, in combination with gemcitabine and cisplatin: a phase I study. Clin Cancer Res 15: 5584-5590, 2009.

15. Tolcher AW, Mita M, Meropol NJ, et al: Phase I pharmacokinetic and biologic correlative study of mapatumumab, a fully human monoclonal antibody with agonist activity to tumor necrosis factor-related apoptosis-inducing ligand receptor-1. J Clin Oncol 25: 1390-1395, 2007.

16. Trarbach T, Moehler M, Heinemann V, et al: Phase II trial of mapatumumab, a fully human agonistic monoclonal antibody that targets and activates the tumour necrosis factor apoptosisinducing ligand receptor-1 (TRAIL-R1), in patients with refractory colorectal cancer. Br J Cancer 102: 506-512, 2010
17. Younes A, Vose JM, Zelenetz AD, et al: A Phase $1 \mathrm{~b} / 2$ trial of mapatumumab in patients with relapsed/refractory non-Hodgkin's lymphoma. Br J Cancer 103: 1783-1787, 2010.

18. Herbst RS, Eckhardt SG, Kurzrock R, et al: Phase I doseescalation study of recombinant human Apo2L/TRAIL, a dual proapoptotic receptor agonist, in patients with advanced cancer. J Clin Oncol 28: 2839-2846, 2010.

19. den Hollander MW, Gietema JA, de Jong S, et al: Translating TRAIL-receptor targeting agents to the clinic. Cancer Lett 332: 194-201, 2013.

20. Van Valen F, Fulda S, Truckenbrod B, et al: Apoptotic responsiveness of the Ewing's sarcoma family of tumours to tumour necrosis factor-related apoptosis-inducing ligand (TRAIL). Int J Cancer 88: 252-259, 2000.

21. Picarda G, Lamoureux F, Geffroy L, et al: Preclinical evidence that use of TRAIL in Ewing's sarcoma and osteosarcoma therapy inhibits tumor growth, prevents osteolysis, and increases animal survival. Clin Cancer Res 16: 2363-2374, 2010.

22. Tomek S, Koestler W, Horak P, et al: Trail-induced apoptosis and interaction with cytotoxic agents in soft tissue sarcoma cell lines. Eur J Cancer 39: 1318-1329, 2003.

23. Kang Z, Chen JJ, Yu Y, et al: Drozitumab, a human antibody to death receptor 5 , has potent antitumor activity against rhabdomyosarcoma with the expression of caspase- 8 predictive of response. Clin Cancer Res 17: 3181-3192, 2011.

24. Kontny HU, Hammerle K, Klein R, Shayan P, Mackall CL and Niemeyer CM: Sensitivity of Ewing's sarcoma to TRAILinduced apoptosis. Cell Death Differ 8: 506-514, 2001.

25. Mitsiades N, Poulaki V, Mitsiades C and Tsokos M: Ewing's sarcoma family tumors are sensitive to tumor necrosis factorrelated apoptosis-inducing ligand and express death receptor 4 and death receptor 5. Cancer Res 61: 2704-2712, 2001.

26. Wu GS, Burns TF, McDonald ER III, et al: KILLER/DR5 is a DNA damage-inducible p53-regulated death receptor gene. Nat Genet 17: 141-143, 1997

27. Pishas KI, Al-Ejeh F, Zinonos I, et al: Nutlin-3a is a potential therapeutic for Ewing sarcoma. Clin Cancer Res 17: 494-504, 2011.

28. Zinonos I, Labrinidis A, Lee M, et al: Apomab, a fully human agonistic antibody to DR5, exhibits potent antitumor activity against primary and metastatic breast cancer. Mol Cancer Ther 8: 2969-2980, 2009.

29. Noll JE, Jeffery J, Al-Ejeh F, et al: Mutant p53 drives multinucleation and invasion through a process that is suppressed by ANKRD11. Oncogene 31: 2836-2848, 2011

30. Neilsen PM, Noll JE, Suetani RJ, et al: Mutant p53 uses p63 as a molecular chaperone to alter gene expression and induce a proinvasive secretome. Oncotarget 12: 1203-1217, 2011.

31. Neilsen PM, Cheney KM, Li CW, et al: Identification of ANKRD11 as a 53 coactivator. J Cell Sci 121: 3541-3552, 2008

32. Suetani RJ, Ho K, Jindal S, et al: A comparison of vitamin D activity in paired non-malignant and malignant human breast tissues. Mol Cell Endocrinol 362: 202-210, 2012.

33. Zhao L, Wientjes MG and Au JL: Evaluation of combination chemotherapy: integration of nonlinear regression, curve shift, isobologram, and combination index analyses. Clin Cancer Res 10: 7994-8004, 2004.

34. Fulda S, Kufer MU, Meyer E, van Valen F, DockhornDworniczak B and Debatin KM: Sensitization for death receptor- or drug-induced apoptosis by re-expression of caspase- 8 through demethylation or gene transfer. Oncogene 20: 5865-5877, 2001 . 